\title{
Potential Candidate Genes and Pathways Affecting the Metabolism of Jaw Fats in Risso's Dolphin: Based on Transcriptomics
}

Jayan Senevirathna ( $\nabla$ duminda@uwu.ac.lk)

University of Tokyo

Ryo Yonezawa

University of Tokyo

Yoji Igarashi

Mie University

Taiki Saka

University of Tokyo

Kazutoshi Yoshitake

University of Tokyo

Shigeharu Kinoshita

University of Tokyo

Noriko Funasaka

Mie University

Shuichi Asakawa

University of Tokyo

\section{Research Article}

Keywords: Dolphin, Echolocation, Acoustic fat, RNA sequencing, Functional enrichments, Lipid metabolism

Posted Date: January 27th, 2021

DOl: https://doi.org/10.21203/rs.3.rs-144280/v1

License: (c) (1) This work is licensed under a Creative Commons Attribution 4.0 International License.

Read Full License 


\section{Abstract}

Jaw fats play a key role in echolocation in toothed whales. These fats are located along the outer and inner segments of the lower jawbone. A ribose nucleic acid (RNA) sequencing technique was employed to investigate transcriptomes of these two types of jaw fat tissues in Risso's dolphins. We identified 1,899 upregulated common genes in both fat tissues. The differentially-expressed genes (DEGs) analysis showed that 34 and nine known genes were significantly upregulated in outer and inner jaw fats, respectively. A functional enrichment analysis was conducted by Enricher; lipid metabolism-related gene ontologies (GO) and pathways were identified $(p<0.05)$. Based on these analyses, $A P O H, H N F 4 A, M Y F 6$, SLC1A2, SLC2A2 and $A L D O B$ were key genes for lipid metabolism in the outer jaw fat which are mainly involved with lipoprotein lipase activities. However, APP, DHX9, PXMP4 and THBS4 genes were highly expressed in the inner jaw fat, and their main functional enrichments were amyloid-beta formation and the activation of ECM-receptor interaction. These recent findings provide evidence for de novo lipid synthesis and as a new concept, the APP may be involved with transferring sound wave signals from the inner jaw fat to the brain via neurons, and further studies are necessary for revealing the puzzle of echolocation in toothed whales.

\section{Introduction}

Sound transmission by adipose tissues in toothed whales (suborder Odontoceti: dolphins, porpoises and whales) is one of the most important macro-evolutionary adaptations to the aquatic environment. Acoustic fats located in the head region, commonly categorized as melon fat and mandibular/jaw fats, play a crucial role in transmitting and receiving sounds, respectively ${ }^{1-3}$. Jaw fats are present at the outer and inner regions of the lower jaw and act as a "jawphone" that hears the returning echoes "; these are also accredited for directing waveguide sounds towards the ear (Aroyan et al., 1992; Koopman et al., 2006a).

This study specially focuses on the acoustic fats of toothed whales, not the blubber fats. It is understood that these fats consist of both triacylglycerol (TAG) and wax esters (WE). The presence of WEs in whales has been investigated by many scientists, however their functions are still unclear. Deposition of wax in the ear plugs of baleen whales has already been recorded ${ }^{7}$, and the accumulation of wax esters in adipose tissues of toothed whales was also investigated ${ }^{2,8,9}$. Generally, scientists believe fatty acids and fatty alcohols that produce wax esters are commonly found. However, acoustic fats of toothed whales contain branched-chain fatty acid (BCFA) such as isobutyl-CoA and isovaleryl-Co-A-derived i-5:0, i-10:0, i$12-0, i-15: 0, i-16: 0$ and $i-16: 0$ alcohol, which is unusual when compared to other mammals ${ }^{2}$. Two common pathways are suggested for the synthesis of these fatty acids like odd-chained BCFA by leucine and even-chained BCFA from valine and isoleucine ${ }^{2}$. Many toothed whale species contain a mixture of TAGs and WEs in their melon and jaw fat deposits. The varied compositions of this mixture depends on many factors in different species and taxonomically 2,10 . 
The compositions of fatty acids and fatty alcohols in the jaw fats of toothed whales are diverse ${ }^{4,11}$. For example, there are different kinds of fatty alcohol accumulations in the mandibular fats of short-finned pilot whales, pygmy sperm whales and several species of beaked whale ${ }^{2}$. A past study ${ }^{12}$ has investigated comparative lipid patterns of melons, jaws and blubber of Risso's dolphin, however there is no detailed investigation of the lipid composition of this species. Jaw fats in different dolphin species have been studied ${ }^{11}$, and the findings identified a differential distribution of fatty acids and alcohols in outer and inner jaw fats. This study observed a large amount of branched-chain fatty alcohols within the inner jaw fat, but their function was not clearly defined. They ${ }^{11}$ suggested toothed whales can biosynthesize these lipid classes due to the diverse array and complexity of their distributions. However, there are no comprehensive studies recorded on the jaw fats of many toothed whales, and its functional mechanism in echolocation still not cleared. Another study ${ }^{3}$ found a difference in the microvascular distributions in both jaw fat tissues in some species; however, these were lower when compared to the blubber fat. One possible reason for this difference is the reduction of gas exchanges and interference of sound pathways.

There are three different types of adipocytes in mammals which show different origin, morphology and gene expression ${ }^{13}$. Adipose tissue plays an important role in mammals for regulating their metabolisms, fatty acid syntheses, adipocytes differentiations and significant wax ester syntheses in toothed whales. The Kennedy pathway is identified as the TAG biosynthesis pathway in bacteria in de novo way, and it is catalyzed by diacylglycerol acyltransferase (DGAT). Acyl-CoA-fatty alcohol acyltransferase (WE synthase, WS) is a catalyzed WE synthesis in bacteria ${ }^{14}$. Several lipid-related studies in bacteria suggest that the complex synthesis of lipids in cells is difficult to understand, and it involves many metabolic pathways and enzymes ${ }^{15}$. Lipid accumulation in outer and inner jaw fat tissues of toothed whales is uncommon and highly diverse. Therefore, to uncover the mystery of lipid synthesis, accumulation and migration in jaw fat tissues in toothed whales, a gene expression analysis must to be conducted.

Ribose nucleic acid (RNA) sequencing is the popular technique for identifying differentially-expressed genes (DEGs) in different tissues that provide some facts about the genetic regulation of lipid metabolism ${ }^{16}$. A transcriptome analysis is appropriate to identify key genes in different jaw tissues that might be closely related to the different fatty acid distributions and can be used to clarify the functions of related metabolic pathways. In this study, we used the Illumina sequencing technique to study transcriptome changes in two different jaw fats of Risso's dolphin in order to uncover the hidden mystery of lipid migration and unusual fatty acid synthesis in toothed whales. Furthermore, several biological processes and pathways in lipid metabolism and echolocation were identified by integrating DEGs in our functional enrichment analysis. This data will serve as a blueprint of gene expression in Risso's dolphins which will also help to clear genes related to fatty acid synthesis pathways in toothed whales. Revealing the lipid metabolism of toothed whales may contribute to the artificial biosynthesis of these important fats. These findings may also help provide protection for these animals in the future.

\section{Materials And Methods}




\section{Sample preparation}

In February 2019, two varieties of adipose tissues in the lower jaw region, the inner jaw fat (IJF) and the outer jaw fat (OJF) were obtained from three male of Risso's dolphins (sample ID: 19TK409, 19TK410 and 19TK411) under the cooperation of the biological surveys by the National Research Institute of Far Seas Fisheries, Japan Fisheries Research and Education Agency. All the samples were quickly preserved in RNA later and then transported to the laboratory and stored at $-80^{\circ} \mathrm{C}$ for total RNA extraction.

\section{RNA isolation, clean-up, quantification and qualification}

Total RNA from the frozen samples was extracted using RNAiso Plus (Takara Bio Inc., Shiga, Japan) following the manufacturer's instructions. RNA clean-up was also conducted using the manufactured protocol of NucleoSpin RNA Clean-up XS of MACHEREY-NAGEL ${ }^{123}$. Purity, concentration and integrity of extracted RNA were determined using Qubit ${ }^{\circledR}$ RNA Assay Kit in Qubit ${ }^{\circledR} 2.0$ Flurometer (Life Technologies, Carlsbad, CA, USA), and an Agilent analysis was conducted by the Agilent Tape Station 2200 system (Agilent Technologies, Santa Clara, CA, USA). The isolated RNA samples were stored in $-80^{\circ} \mathrm{C}$ for further downstream applications.

\section{cDNA library construction and sequencing}

Library sequences were generated using low sample protocol of Trueseq Stranded mRNA sample preparation guide for Illumina ${ }^{\circledR}$ Hiseq (Illumina, San Diego, CA, USA) following manufacturer's recommendations. Poly-A containing mRNA was purified by poly-T oligo attached to magnetic beads, fragmented and primed for CDNA synthesis. Cleaved RNA was reverse transcribed and primed with random hexamers into first-strand CDNA using reverse transcriptase and random primers. dUTP incorporated dTTP replacement strand was made to generate ds CDNA, and AMPure XP beads were used to separate blunt-ended double-strand (ds) cDNA. A single ' $A$ ' nucleotide was added to the 3 ' ends of ds cDNA fragments, and multiple indexing RNA adapters were ligated.

Library DNA that had adapter molecules in both ends were amplified by the PCR. These libraries were used for the quality control analysis and quantification by Agilent Tape Station 2200 system (Agilent Technologies, Santa Clara, CA, USA). Finally, these libraries were normalized and pooled to a single library. The library was loaded into a flow cell for clustering with surface-bound oligos complementary to the library adapters and bridge amplification was done to prepare clonal clusters for sequencing by Illumina HiSeq platform (Illumina, San Diego, CA, USA), and paired-end raw reads were generated by RTA2 and bcl2fastq2-v2-20.0 (Macrogen NGS service).

\section{Quality assessment and mapping to a reference genome}

To obtain high-quality clean reads, raw reads were used to trim adapters and filtered by removing the poly-A sequence that contained more than five bases at the $3^{\prime}$ end and whose Phred quality (Q) scores $\leq$ 20. Then, clean reads were mapped to the reference common bottlenose dolphin (Tursiops truncates) 
genome sequence (GCF_001922835.1_NIST_Tur_tru_v1, downloaded from NCBI) ${ }^{124}$, filtered by PRINSEQ 0.20.4 ${ }^{125}$, aligned by HISAT2 2.1.0 ${ }^{126}$ and annotated to the GTF of same genome by StringTie 1.3.4 ${ }^{127}$ in the RNA-seq analysis PortablePipeline-win-v0.9c 2019 (https://github.com/c2997108/OpenPortablePipeline). Cufflink was used for mapped read splicing, and reads were then compared with the genomic annotation information to explore new genes ${ }^{128}$. Additionally, the number of reads mapped to a particular gene were calculated using a ballgown algorithm ${ }^{129}$

(https://www.bioconductor.org/packages/release/bioc/vignettes/ballgown/inst/doc/ballgown.html) in the pipeline. Finally, the gene count data and normalized fragments per kilobase of exon per million fragments mapped (FPKM) values were obtained.

\section{Differential expression analysis and gene functional annotation}

The expression correlation of IJF and OJF of three individual animals and multi-dimensional scaling (MDS) was performed with R package using expressed genes. DEGs between two libraries were identified by the R packages, edgeR (http://bioconductor.jp/packages/release/bioc/html/edgeR.html) ${ }^{130}$ and Cuffdiff (https://bioinformaticshome.com/tools/rna-seq/descriptions/Cuffdiff-2.html) ${ }^{131}$. Genes with $\mid \log 2$ (fold change) $\mid \geq 1$ and $p$-value $<0.05$ and an adjusted $p$-value $<0.05$ were regarded as DEGs. Also, resulting $\mathrm{p}$ values were adjusted by Benjamini and Hochberg's approach for controlling the False Discovery Rate (FDR). To further visualize the DEGs of IJFs and OJFs, a heatmap was generated using the $R$ package of pheatmap using the FPKM values of DEGs.

In addition, the DEGs detected by edgeR and Cuffdiff were subjected to a functional enrichment analysis, including a gene ontology (GO) and a pathway enrichment analysis using the Enrichr online version (https://amp.pharm.mssm.edu/Enrichr/) ${ }^{132}$. GO terms and pathways with q-value $<0.05$ were considered to be significantly enriched. Genes, involved in the significantly enriched GO terms and pathways related to lipid metabolism and fat cell differentiation, were considered as key genes affecting jaw fat metabolism in toothed whales. The identified DEGs were uploaded to GeneMANIA (https://genemania.org/), and gene interactive networks were produced. GeneMANIA has also supplied the genes involved with different functions and FDR values $(<0.05)$ to identify significant GO terms ${ }^{133}$.

\section{Real-Time Quantitative PCR (RT-qPCR) validation}

Total RNAs was extracted from the same selected samples by RNAiso Plus ${ }^{134}$. Approximately 1 ug of total RNA from six samples were used to prepare first strand cDNA by PrimeScript RT master mix kit (Perfect Real Time). Six genes were used as the interest genes with normalizing by FTL, a newly found housekeeping gene for these tissues as a reference to validate all samples (unpublished data). The amplification was performed in 96-well plates in an ABI 7300 Fast Real-Time PCR system (ABI, Alameda, CA, USA) using the SYBR Premix Ex Taq ${ }^{\text {TM }}$ Takara kit ${ }^{135}$. One $q P C R$ reaction condition was as follows: $10 \mathrm{ul}$ SYBR Premix Ex Taq (1x), 0.8 ul of $50 \mathrm{nM}$ of both Forward and Reverse primers, $0.4 \mathrm{ul}$ of ROX 
reference dye (50X), $6 \mathrm{ml}$ of nuclease-free water and $2 \mathrm{ul}$ of $100 \mathrm{ng} \mathrm{cDNA}$ in a $20 \mathrm{~mL}$ total volume. Three biological replicates were tested, and relative gene expression was analyzed by the delta delta $\mathrm{Ct}$ method.

\section{Results}

\section{Illumina sequencing}

In this study, a paired-end RNA-seq approach was conducted to investigate the transcriptome in IJF and OJF tissues in three Risso's dolphins. First, six cDNA libraries were constructed and sequenced, and 4.195 Gb clean reads were obtained. More than $92.38 \%$ of the data yielded a high-quality score (Q20) (S1 table). GC content from these clean data reached more than $49.71 \%$. Then, all raw reads were mapped onto the genome of common bottlenose dolphin and produced total of clean reads ranged from 693,422$3,888,391$, and an additional investigation was conducted in a pipeline analysis.

The expressions of mRNAs in six libraries were evaluated by their FPKM values. The expression levels of genes ranged from 0 to 240,973.7 FPKM. Based on the FPKM values, the distribution of upregulated genes was determined among two different jaw fat tissues (Figure 1, A). Commonly in both jaw fat tissues, 1,899 genes were found with equal or more than 1 FPKM. Moreover, IJF contained more upregulated unique genes than the OJF. The MDS plot (Figure 1, B), showing a variation among RNA-seq libraries based on log10 distribution, resulted in the suggested clustering of samples as IJF and OJF. In addition, Figure 1, $\mathrm{C}$ also showed how the two jaw fats had more overlapping genes. Raw gene counts, Figure 1, D, indicated that IJF contained more gene counts than the OJF (S1 table, S2 table).

\section{Differential expression analysis}

Differential gene expression was performed in this study to identify transcriptional variations occurring between the IJFs and OJFs. DEGs were detected in two statistical methods for high accuracy, edgeR and Cuffdiff. The edgeR found a group of five genes upregulated in IJF and 21 genes in OJF (FDR < 0.05$)$. The Cuffdiff results also revealed four and 13 DEGs in IJFs and OJFs, respectively (q value $<0.05$ ) (Figure 2, S2 table and S3 table). Among all the genes annotated to the common bottlenose dolphin genome, highly-expressed genes in both fat tissues were summarized in the heatmap, Figure 2, C. In both analyses, the albumin (ALBU) gene was identified as significantly expressed in OJF.

\section{Functional enrichment analyses of DEGs in both jaw fats}

GO and pathway analyses were conducted using DEGs to reveal the potential functional categories in IJF and OJF, separately, by the Enricher online tool. In the OJF, 71 GO biological processes, six GO cellular components, $14 \mathrm{GO}$ molecular functions and 23 Jensen compartments were classified (S4 table). The IJF was exhibited $42 \mathrm{GO}$ biological processes, $19 \mathrm{GO}$ cellular components, $33 \mathrm{GO}$ molecular functions and 72 Jensen compartments (S5 table). The DEGs were enriched in different signaling pathways, and the analysis-identified pathways were based on several of the following databases. For OJF, two by KEGG 2019 human, 29 MGI-Mammalian, 13 by BioPlanet 2019, seven by WikiPathways 2019 (S6 table). For IJF, 
two by KEGG 2019 human, 48 by MGI-Mammalian, 17 by BioPlanet 2019, six by WikiPathways 2019 (S7 table). Based on enrichment results, we were interested in lipid metabolism related GO and pathways. Therefore, those significantly enriched GO and pathways terms for OJF and IJF were emphasized in Tables 1 and 2, respectively, for further discussion.

In the OJF, APOH, HNF4A, MYF6, SLC1A2, SLC2A2 and ALDOB were noteworthy as hub genes for lipid metabolisms related enrichment terms like, positive regulation of lipoprotein and triglyceride lipase activity, glycolysis and pentose phosphate pathway. The genes like APP, DHX9, PXMP4 and THBS4 were highlighted in the IJF which involved with lipid metabolism, transport and localization activities.

Table 1. Lipid metabolism related functional enrichments in OJF using Enricher results 


\begin{tabular}{|c|c|c|c|}
\hline Enrichment term & Category & Genes & $\begin{array}{l}\text { P- } \\
\text { value }\end{array}$ \\
\hline phospholipid homeostasis (G0:0055091) & Biological & HNF4A & 0.0127 \\
\hline $\begin{array}{l}\text { positive regulation of lipoprotein lipase activity } \\
\text { (G0:0051006) }\end{array}$ & Biological & $\mathrm{APOH}$ & 0.0127 \\
\hline $\begin{array}{l}\text { positive regulation of triglyceride lipase activity } \\
\text { (G0:0061365) }\end{array}$ & Biological & $\mathrm{APOH}$ & 0.0143 \\
\hline $\begin{array}{l}\text { regulation of cholesterol homeostasis } \\
\text { (GO:2000188) }\end{array}$ & Biological & HNF4A & 0.0190 \\
\hline $\begin{array}{l}\text { regulation of lipoprotein lipase activity } \\
(\mathrm{GO}: 0051004)\end{array}$ & Biological & $\mathrm{APOH}$ & 0.0315 \\
\hline acylglycerol homeostasis (GO:0055090) & Biological & HNF4A & 0.0346 \\
\hline glycolytic process (G0:0006096) & Biological & ALDOB & 0.0361 \\
\hline triglyceride homeostasis (G0:0070328) & Biological & HNF4A & 0.0438 \\
\hline very-low-density lipoprotein particle (G0:0034361) & Cellular & $\mathrm{APOH}$ & 0.0237 \\
\hline lipase activator activity (GO:0060229) & Molecular & $\mathrm{APOH}$ & 0.0111 \\
\hline triglyceride-rich lipoprotein particle & Jensen & $\mathrm{APOH}$ & 0.0299 \\
\hline very-low-density lipoprotein particle & Jensen & $\mathrm{APOH}$ & 0.0299 \\
\hline high-density lipoprotein particle & Jensen & $\mathrm{APOH}$ & 0.0408 \\
\hline $\begin{array}{l}\text { Pentose phosphate pathway } \\
\text { (fatty acid synthesis) }\end{array}$ & KEGG/BioPlanet & ALDOB & 0.0469 \\
\hline HNF3B pathway (fat metabolism) & Bioplanet & $\begin{array}{l}\text { HNF4A, SLC2A2, } \\
\text { ALDOB }\end{array}$ & $\begin{array}{l}5.04 \mathrm{E}- \\
05\end{array}$ \\
\hline Glycolysis and gluconeogenesis & BioPlanet & SLC2A2, ALDOB & 0.0064 \\
\hline $\begin{array}{l}\text { Facilitative sodium-independent glucose } \\
\text { transporters }\end{array}$ & BioPlanet & SLC2A2 & 0.0190 \\
\hline $\begin{array}{l}\text { Developmental biology } \\
\text { (white adipocyte differentiation) }\end{array}$ & BioPlanet & $\begin{array}{l}\text { HNF4A, MYF6, } \\
\text { SLC2A2 }\end{array}$ & 0.0291 \\
\hline Glutathione conjugation & BioPlanet & GSTA2 & 0.0331 \\
\hline Glycolysis & BioPlanet & ALDOB & 0.0423 \\
\hline Gluconeogenesis & BioPlanet & ALDOB & 0.0484 \\
\hline Glycolysis and Gluconeogenesis WP534 & WikiPathways & SLC2A2, ALDOB & 0.0023 \\
\hline Cori Cycle WP1946 & WikiPathways & SLC2A2 & 0.0253 \\
\hline
\end{tabular}


Table 2. Lipid metabolism related GO enrichments in IJF using Enricher results 


\begin{tabular}{|c|c|c|c|}
\hline Enrichment term & Category & Genes & $\begin{array}{l}\mathrm{P}- \\
\text { value }\end{array}$ \\
\hline positive regulation of binding (G0:0051099) & Biological & $\begin{array}{l}\text { APP, } \\
\text { DHX9 }\end{array}$ & $\begin{array}{l}3.36 \mathrm{E}- \\
04\end{array}$ \\
\hline $\begin{array}{l}\text { positive regulation of amyloid-beta formation } \\
\text { (GO:1902004) }\end{array}$ & Biological & APP & 0.0035 \\
\hline pyroptosis (G0:0070269) & Biological & $\mathrm{DHX} 9$ & 0.0035 \\
\hline transport along microtubule (G0:0010970) & Biological & APP & 0.0052 \\
\hline $\begin{array}{l}\text { positive regulation of lymphocyte migration } \\
\text { (GO:2000403) }\end{array}$ & Biological & APP & 0.0059 \\
\hline regulation of T cell migration (G0:2000404) & Biological & APP & 0.0059 \\
\hline positive regulation of T cell migration (GO:2000406) & Biological & APP & 0.0070 \\
\hline $\begin{array}{l}\text { positive regulation of nucleocytoplasmic transport } \\
(\text { GO:0046824) }\end{array}$ & Biological & DHX9 & 0.0115 \\
\hline glial cell development (G0:0021782) & Biological/Jensen & APP & 0.0070 \\
\hline regulation of cation channel activity (GO:2001257) & Biological & APP & 0.0246 \\
\hline protein localization to organelle (G0:0033365) & Biological & DHX9 & 0.0335 \\
\hline micro-ribonucleoprotein complex (G0:0035068) & Cellular & DHX9 & 0.0024 \\
\hline RISC-loading complex (G0:0070578) & Cellular & DHX9 & 0.0024 \\
\hline peroxisome (G0:0005777) & Cellular & PXMP4 & 0.0318 \\
\hline microbody (G0:0042579) & Cellular & PXMP4 & 0.0318 \\
\hline Golgi lumen (G0:0005796) & Cellular & APP & 0.0338 \\
\hline membrane raft (GO:0045121) & Cellular & APP & 0.0409 \\
\hline axon (G0:0030424) & Cellular & APP & 0.0483 \\
\hline integrin binding (G0:0005178) & Molecular & THBS4 & 0.0324 \\
\hline Nuclear envelope lumen & Jensen & APP & 0.0024 \\
\hline Ciliary rootlet & Jensen & APP & 0.0024 \\
\hline ECM-receptor interaction & KEGG & THBS4 & 0.0283 \\
\hline Peroxisome & KEGG & PXMP4 & 0.0287 \\
\hline Serotonergic synapse & KEGG & APP & 0.0389 \\
\hline Copper homeostasis WP3286 & WikiPathways & APP & 0.0181 \\
\hline Advanced glycosylation end product receptor signaling & BioPlanet & APP & 0.0045 \\
\hline
\end{tabular}


Furthermore, the DEGs related to lipid metabolism were used in GeneMANIA for gene-gene interaction network analysis. In both jaw fat types, key genes were localized and combined with other genes, indicating their close proximity and functional relationships (Figure 3). Compared to human genomes, the PXMP4, DHX9, THBS4 and APP genes were identified as key genes for developing the networks: $67.64 \%$ was physical interactions, $13.50 \%$ was co-expressions, $6.35 \%$ was predicted, $6.17 \%$ was co-localizations, $4.35 \%$ was pathways, $1.40 \%$ genetic interactions and $0.59 \%$ was shared protein domains. In the same manner, key genes in OJF were identified as APOH, HNF4A, MYF6, GSTA2, ALDOB and SLC2A2. These genes were involved to make the networks by $66.41 \%$ of pathways, $25.28 \%$ of co-localizations, $8.02 \%$ of co-expressions and $0.29 \%$ of genetic interactions.

We also checked the expression levels of identified genes in KEGG TAG/WAX synthesis pathways, specially focusing on fatty acid synthesis pathways, valine, leucine and isoleucine biosynthesis pathways and valine, leucine and isoleucine degradation pathways (Table 3 ).

Table 3. Expression of TAG/WAX synthesis genes in three different KEGG pathways in inner and outer jaw fats 


\begin{tabular}{|c|c|c|c|c|}
\hline Gene ID & Gene Name & Relative pathway, Database & $\begin{array}{l}\text { Outer } \\
\text { jaw } \\
\text { FPKM }\end{array}$ & $\begin{array}{l}\text { Inner } \\
\text { jaw } \\
\text { FPKM }\end{array}$ \\
\hline FASN & Fatty Acid Synthase & \multirow[t]{8}{*}{ Fatty acid synthesis, KEGG } & 0 & 7.09 \\
\hline FabD & $\begin{array}{l}\text { Malonyl CoA-acyl carrier protein } \\
\text { transacylase }\end{array}$ & & 0 & 0.9 \\
\hline FABP5 & Fatty acid binding protein & & 193.92 & 87.53 \\
\hline $\mathrm{ACP}$ & Acyl carrier protein & & 118.07 & 87.80 \\
\hline CBR4 & $\begin{array}{l}\text { Carbonyl reductase family } \\
\text { member } 4\end{array}$ & & 0 & 2.9 \\
\hline HSD17B8 & $\begin{array}{l}\text { Hydroxysteroid 17-Beta } \\
\text { Dehydrogenase } 8\end{array}$ & & 0 & 3.68 \\
\hline HTD2 & $\begin{array}{l}\text { Hydroxyacyl-thioester dehydratase } \\
\text { type } 2\end{array}$ & & 0 & 1.2 \\
\hline MCAT & $\begin{array}{l}\text { Malonyl CoA-acyl carrier protein } \\
\text { transacylase }\end{array}$ & & 104 & 290 \\
\hline BCAT2 & $\begin{array}{l}\text { branched-chain amino acid } \\
\text { aminotransferase/transaminase }\end{array}$ & \multirow[t]{3}{*}{$\begin{array}{l}\text { Valine, leucine and isoleucine } \\
\text { biosynthesis pathway, KEGG }\end{array}$} & 12.75 & 20.23 \\
\hline SDS3 & serine dehydratase & & 80.08 & 0 \\
\hline ilvl & $\begin{array}{l}\text { acetolactate synthase I/II/III large } \\
\text { subunit }\end{array}$ & & 9.3 & 17.98 \\
\hline BCAT2 & $\begin{array}{l}\text { branched-chain amino acid } \\
\text { aminotransferase/transaminase }\end{array}$ & \multirow[t]{3}{*}{$\begin{array}{l}\text { Valine, leucine and isoleucine } \\
\text { degradation pathway, KEGG }\end{array}$} & 12.75 & 20.23 \\
\hline ALDH2 & aldehyde dehydrogenase & & 55.43 & 37.61 \\
\hline WS/DGAT & $\begin{array}{l}\text { Wax ester synthase/acyl- } \\
\text { coenzyme A: diacylglycerol } \\
\text { acyltransferase }\end{array}$ & & 8.9 & 17.41 \\
\hline
\end{tabular}

\section{Validation of results of RNA-seq}

To validate RNA-seq expression, six genes were randomly selected from both jaw fats. The selected genes were APOH, ALDOB and ALBU for the outer fat and APP, PXMP4 and DHX9 for the inner fat. The qPCR analysis was conducted for the same sample replicates. Based on the qPCR data and Ct values, fold gene expressions were calculated for each replicate of two tissue types. The qPCR analysis confirmed that the selected genes were differentially expressed among two different fat tissues, indicating that RNA-seq results were correct and reproducible (Figure 4).

\section{Discussion}


In this study, the jaw fat transcriptome data was obtained from OJFs and IJFs of three male Risso's dolphins for minimum statistical limitations and analyzed by high throughput NGS technology to understand the genetic lineages of metabolism of these specialized acoustic fats. We believe that identifying the fat metabolism of these important animals may contribute industrial applications for biosynthesis and protecting aquatic wildlife.

We first identified the DEGs in both jaw fat tissues based on total transcriptome data and analyzed if for functional annotation. Illumina produce short readings with an average length of $500 \mathrm{bp}$ in large numbers of copies which are annotated to the reference genome to identify genes. However, RNA-seq technology is still being developed in order to perform long-read sequencing ${ }^{17}$. Three biological replicates have used to optimize transcriptome data, and edgeR was used as a differential expression tool for false positive performances, as recommended ${ }^{18,19}$. High quality and accurate sequenced data were obtained from this study for further analysis. Cuffdiff is also a supportive tool for assembling readings from two or more biological conditions and identify DEGs for transcriptional analyses ${ }^{20}$.

The two jaw fats of toothed whales, IJF and OJF, showed several differences based on previous studies $8,21-23$. The OJF is located around the lower jaw of toothed whales and directly connected with other tissues; however, IJF is present inside the lower jaw hole. Therefore, the mechanism of generation for these two fats is certainly a concept worthy of study. According to the previous findings, there is a phylogenetic influence for the development of these acoustic fats and endogenous synthesis 21,24 . Additional studies have emphasized that sound transmission through the fat bodies is possible and may alter the wavelength physically and change depending on the species, thereby reducing competition over predation. However, detailed studies are still needed to explain this unknown puzzle. We also recorded differences in upregulated DEGs by bioinformatics tools in both of the fat tissue types; to our knowledge, this observation is the first of its kind. These findings indicate that those DEGs are related to lipid metabolism activities. A higher number of DEGs were observed in the outer fat compared to the inner fat.

Interestingly, fatty acid binding protein $1(F A B P 1)$, apolipoprotein $\mathrm{H}(A P O H)$, fructose-bisphosphate aldolase $\mathrm{B}(A L D O B)$, zinc finger protein 37A (ZN37A), ALBU, solute carrier families 1 and 2 (SLC1A2 \& $S L C 2 A 2)$ and hepatocyte nuclear factor 4, alpha (HNF4A) were identified in the OJF, which may directly engage with lipid metabolism. A study has revealed the FABP4 is associated with fat deposition in bovine populations ${ }^{25}$. The FABP1 is a highly-expressed DEG in the OJF and downregulated in IJF in the current study. The $A P O H$ protein is involved with triggering of lipoprotein lipase in lipid metabolism ${ }^{26,27}$. The $A \angle D O B$ gene is an important gene in mammals' metabolisms and homeostasis. This gene contributes to fructose metabolism and, therefore, enhances glycogen and lipid synthesis ${ }^{28}$. $Z N 37 A$ is one of zinc finger proteins (ZFPs), a direct study on this lipid's metabolism has not been found, however ZFPs are involved with lipid bindings and lipid and glucose metabolism ${ }^{29,30}$. ALBUis also identified as a biomarker for lipid-related diseases in human-like obesity ${ }^{27,31}$. SLC1A2 and SLC2A2 are solute carrier associated proteins which are involved in lipid biosynthesis, fatty acid metabolic process and fatty acid transport ${ }^{32-35}$. HNF4A is a transcription factor that binds with fatty acids ${ }^{36}$, regulates the expression of 
apolipoproteins ${ }^{37}$ and is involved with lipid metabolism ${ }^{38}$. On the other hand, the IJF contains DEGs of amyloid beta precursor protein (APP), Thrombospondin-4 (TSP4), mRNA decay activator protein (ZFP36L 1) and ATP-dependent RNA helicase A (DHX9). These genes share some relationships with lipid metabolism such as the APP and TSP4 genes which are involved with bone marrow adiposity ${ }^{39-41}$; $D H X 9$ shows an oxidative stress responsiveness ${ }^{42}$. Another study has identified a complex bidirectional link between lipids and $A P P$ related to lipid alteration, $A P P$ processing and regulation of lipid metabolism pathways in brains ${ }^{43}$.

We also checked the expression levels of common genes of TAG/WAX metabolism KEGG pathways (Table 4) to acquire a comprehensive biological insight from the enrichment of expressed genes. Interestingly, FAS, malonyl CoA-acyl carrier protein transacylase $(F a b D)$, carbonyl reductase family member 4 (CBR4), hydroxysteroid 17-Beta Dehydrogenase 8 (HSD17B8), hydroxyacyl-thioester dehydratase type 2 (HTD2), malonyl CoA-acyl carrier protein transacylase (MCAT) of the fatty acid synthesis, branched-chain amino acid aminotransferase/transaminase (BCAT2), acetolactate synthase I/II/III large subunit (ilVI) of the valine, leucine and isoleucine biosynthesis pathway, BCAT2 and wax ester synthase/acyl-coenzyme A: diacylglycerol acyltransferase (WS/DGAT) of the valine, leucine and isoleucine degradation pathways were more expressed in the IJF than the OJF. However, fatty acid binding protein (FABP5) and acyl carrier protein $(A C P)$, of fatty acid synthesis pathway and aldehyde dehydrogenase $(A L D H 2)$ of the valine, leucine and isoleucine degradation pathways, were increasingly expressed in the OJF compared with the IJF.

Based on this diversity of DEGs in both jaw fat tissues, we suggest that there is a clear possibility of endogenous synthesis of these specialized fats in particular locations of the lower jaw area of toothed whales. Therefore, we concur with the previous suggestion of previous experts (Koopman, 2018): The evolution of unique de novo lipid biosynthesis pathways to are used produce wax esters and short branched-chain pathways in the suborder Odontoceti (toothed whales).

In this study, we used Enrichr, the online tool of the Ma'ayan lab, mainly for the functional enrichment analysis using the DEG sets in the two tissue types. The specialties of this tool are a large collection of diverse gene libraries, improved programming interface and a variety of visualization choices, fuzzy enrichments, easy to use, open source and ranking enriched terms with $p$ value ${ }^{44,45}$. The functional enrichment analysis contains two categories such as a GO analysis and a pathway analysis, both of which were conducted using Enrichr. GOs were comprised with several groups, however we considered four main categories such as biological, cellular, molecular and Jensen. Pathway enrichments were also categorized into several groups. In this study, we used KEGG 2019 human, MGI-Mammalian, BioPlanet 2019 and WikiPathways 2019 to identify reliable pathways. The selection of an updated database for functional enrichments is highly advised ${ }^{46}$; therefore, we believed a collection of information from several database may provide accurate results. We mainly focused 23 terms related to the lipid metabolisms for further discussions to prove our hypothesis. 
In the OJF (Table 1), the main lipid related identified biological processes were phospholipid homeostasis (GO:0055091), positive regulation of lipoprotein lipase activity (G0:0051006), positive regulation of triglyceride lipase activity (GO:0061365), regulation of cholesterol homeostasis (G0:2000188), regulation of lipoprotein lipase activity (G0:0051004), acylglycerol homeostasis (G0:0055090), glycolytic process (GO:0006096) and triglyceride homeostasis (G0:0070328). Phospholipid homeostasis is involved with regulation of phospholipids in organisms and specially in mammals, it can increase curvature elastic stress which may contribute to de novo lipid synthesis ${ }^{47}$. The positive regulation of lipoprotein lipase activity is also relatively observed in this tissue. This supports the hydrolysis of triacylglycerol and the uptake of free fatty acids from the plasma ${ }^{48}$. The activities to increase triglyceride lipase in OJF has also enriched. Angiopoietin-like proteins, circulating triglyceride (TG) levels ${ }^{49}$ and peroxisome proliferatoractivated receptors (PPAR-gama) can regulate adipose triglyceride lipase in adipocytes ${ }^{50}$. Cholesterol homeostasis and fatty acid metabolism can be regulated by liver $X$ receptors (LXR), and PPAR-gama can also induce expression of ATP binding cassette transporters (ABCA) which is essential for lipid raft maintenance and apolipoproteins by upregulating $L X R^{51,52}$. Multifunctional 0 -acyltransferase can catalyze the acylglycerol homeostasis, waxes and retinyl esters which are important in lipid metabolisms 53 . The overexpression of acylglycerol, which catalyze triacylglycerol formation ${ }^{54}$. Glycolytic process triggers glycolytic flux which regulates the fatty acyl-CoAs ${ }^{55}$. Triglyceride homeostasis is the most important enriched ontology in this tissue, which is directly relate with the lipid metabolism. Triglycerides and cholesterol esters can store in lipid droplets and can contribute to de novo lipid synthesis ${ }^{56}$. The triglyceride-rich lipoprotein particle is also enriched in the Jensen compartments. The lipid metabolismrelated GO cellular component, a very-low-density lipoprotein (VLDL) particle (G0:0034361), was enriched in the OJF. This is also highlighted in the Jensen compartments. VLDLs are composed of triacylglycerols and cholesterols which are produced from liver and travel via plasma and can hydrolyze to provide fatty acids in a particular organ ${ }^{57,58}$. In relation to the VLDLs, high-density lipoprotein particles are also enriched according to the Jensen compartments. The lipase activator activity (G0:0060229) is the only enriched molecular GO term in the OJF, whereas lipoprotein lipase is highly important for lipid metabolism in animals ${ }^{59}$.

Pentose phosphate pathway, hepatocyte nuclear factor 3-beta (HNF3B) pathway, glycolysis and gluconeogenesis, facilitative sodium-independent glucose transporters, developmental biology (white adipocyte differentiation), glutathione conjugation, glycolysis, gluconeogenesis, glycolysis and gluconeogenesis WP534 and Cori Cycle WP1946 were the enriched terms in OJF according to KEGG, BioPlanet and WikiPathways. A study has revealed that an oxidative pentose phosphate pathway can doubly enhance fatty acid biosynthesis ${ }^{60}$, and this pathway can provide $\mathrm{NADH}$ to accelerate the production of acetyl-CoA, a precursor of fatty acids ${ }^{61}$. Acceleration of an HNF3B pathway can activate genes related to glucose homeostasis, therefore indirectly influencing lipid metabolism ${ }^{62}$. Glycolysis and gluconeogenesis are other enriched pathways that are involved in synthesizing lipids; fatty acid metabolism may contribute to de novo fatty acid synthesis ${ }^{63}$. Facilitative sodium-independent glucose transporters are an active pathway in OJF, they are responsible for the absorption and distribution of 
glucose and other sugars in mammals ${ }^{64}$. Moreover, BioPlanet database has identified the white adipocyte differentiation in the nuclear receptor PPAR-gama may play an important role in this activity (Niemala et al., 2008; Mueller, 2014). The glutathione conjugation pathways also has a strong affinity for glutathione-derivatized fatty acids ${ }^{67}$. The Cori cycle is also involved within the gluconeogenesis, therefore it may be responsible for the metabolism in the liver and related to lipolysis in adipose tissues 68 .

In IJF, lipid metabolism-related GO enrichment biological terms are shown in the Table 2. The positive regulation of lipid-binding terms comes under the positive regulation of binding; therefore, it is associated with lipid metabolism. ALBP (adipocyte lipid binding protein) includes an intracellular lipid binding family ${ }^{69}$ which is involved in delivering fatty acids to appropriate sites for energy and regulatory processes 70 . The $A P P$ is an important gene found in IJF that is responsible for many functional enrichment terms, especially positive regulation of amyloid-beta formation. Amyloid-beta is directly involved with lipid homeostasis, and many lipids are involved with amyloid-beta lipid regulatory system bidirectionally ${ }^{71}$. Serum amyloid A3 (SAA3) is also an example that was recently found which contributes to lipid deposition in mammals ${ }^{72}$. Also, according to another recent finding, plasma lipids and plasma amyloidbeta has a functional relationship ${ }^{73}$. Activation of pyroptosis is supported with monounsaturated oleic acid and lipotoxicity ${ }^{74}$ and upregulated adipogenesis and misbalanced fatty acid metabolism ${ }^{75}$. Transport along microtubule is also enriched in IJF. In many cells, lipid droplets get active movement through microtubules for various purposes like biogenesis ${ }^{76}$. Microtubules play an important role in the cell to bidirectional transport of cellular cargos ${ }^{77}$. Positive regulation of lymphocyte migration is another enriched term, for example, immune cells interact with lipids and control plasticity and T lymphocytes ${ }^{78}$. Cytotoxic T lymphocytes has relation with maintaining high level of proteins and lipid synthesis ${ }^{79}$. Positive regulation of $\mathrm{T}$ cell migration is also effective on lipid metabolism such as glycolysis, fatty acid and glycosphingolipid metabolism ${ }^{80}$. Positive regulation of nucleocytoplasmic transport in mesenchymal stem cells may involve with adipocyte generation ${ }^{81}$. Glial cell development has enriched in IJF, these cells can accumulate of lipid droplets ${ }^{82}$. Another study has revealed that lipid-mediated communication between glial cells and neurons, however lipid metabolisms in glial cells is still not clear 83,84. The IJF is known as the receiver of echolocated sound waves, therefore cell-cell communication might be a good evidence for further investigations. Moreover, lipoprotein-dependent lipid accumulation may have relation with the origin of glial cell formation in vertebrates ${ }^{85}$. Lipids in cells can regulate cation channel activity in different ways such as fatty acids change membrane mechanics, regulate, interact and modify ion channel functions ${ }^{86}$. Protein localization to organelle was a GO-enriched term in IJF. Phospholipids are important transporters of lipids from endoplasmic reticulum to other organelles ${ }^{87}$. On the other hand, transport can vary significantly with the type of the cell and some organelles have lipids to go along with unique proteomes ${ }^{88}$. Peroxisome is a cellular enriched term in IJF which is direct nexus for lipid synthesis, fatty acid oxidation, production of ether lipids and cellular signaling 89,90 . Furthermore, lipid droplets and peroxisomes maintain lipid homeostasis in cells ${ }^{91,92}$. Microbodies are 
associated with lipid globules and make microbody-lipid globule complex ${ }^{93}$. Lipid metabolism is integrated with Golgi function, membrane raft and control cellular trafficking ${ }^{94,95}$. Axon is another important cellular enriched term in the IJF. Axon can be involved with lipid transportation intracellularly or intercellularly and axon to myelin, however its pathways are still unknown ${ }^{96}$. Lipids are also involved with the regeneration and elongation of axons ${ }^{97-99}$. Integrin binding is the only enriched molecular term of the IJF. The integrin function can be regulated by lipid rafts ${ }^{100}$. Nuclear envelope lumen is the region in between two lipid bilayers, which maintains traffic between the nucleus and cytoplasm ${ }^{101}$, and it is involved with lipid metabolisms ${ }^{102}$. A study has identified that inner nuclear membrane helps to generate nuclear lipid droplets ${ }^{103}$. Cilia are also involve with protein localization in the cell, and lipid modifications properly direct these proteins ${ }^{104}$.

ECM-receptor interaction, peroxisome, serotonergic synapse, copper homeostasis and advanced glycosylation end-product receptor signaling are the main enriched pathways in the IJFs. The extracellular matrix (ECM) receptor interaction is involved with lipid metabolism, adipogenesis and maintain tissue architecture ${ }^{105}$. Integrins play an important role between cells and ECM while significant enrichment has observed in subcutaneous and intramuscular fats in cattle ${ }^{106,107}$. This pathway also recorded as enriched in intramuscular fat metabolism between breast and thigh tissues of chickens ${ }^{108}$, metabolism of fats in tails of sheep ${ }^{109}$. Peroxisomes are also enriched KEGG pathway in IJFs and it is also enriched as a cellular GO term as we described in the earlier paragraph. However, we can further confirm that as a key metabolic organelle, peroxisomes are involved with lipid metabolism for betaoxidation of fatty acids and synthesis of myelin sheath lipids ${ }^{110}$. Serotonergic synapse is a enriched KEGG pathway in this tissue that express the function of lipids as a neurotransmitter ${ }^{111,112}$ and controlling metabolic homeostasis ${ }^{113}$. Copper homeostasis WP3286 is a enriched term from WikiPathways that refers to the IJF, clearly saying that importance of copper for the lipid synthesis and insufficient copper may cause for lipid related diseases ${ }^{114}$. Copper also play a role as a modifier in lipid metabolism and adipocytes require copper to balance the metabolic fuels and de novo lipogenesis ${ }^{115}$. Advanced glycosylation end-product receptor signaling pathways have some correlations with inflammation ${ }^{116}$, cellular signaling ${ }^{117}$ and adipogenesis ${ }^{118}$.

Comparing all the enriched DEGs and functional terms between inner and OJFs, we predict that two different lipid synthesis and metabolism patterns have developed in inner and OJFs in toothed whales. According to the expression levels of common genes in KEGG TAG/WAX synthesis pathways (Table 4), we can clearly identify that valine, leucine and isoleucine biosynthesis and degradation can occur in both fat tissues. The normal fatty acid synthesis also can happen in the inner fat with presence of all genes in the fatty acid synthesis KEGG pathway, but it may not manifest in the OJF. The presence of wax ester in mammalian adipose tissues is not common and may be specified only to toothed whales. The intake of wax esters from diets is impossible, therefore it was strongly suggested that wax in toothed whales should be de novo synthesis endogenously ${ }^{6}$. By accepting this suggestion, our study presents a wax ester synthesis gene, WS/ DGAT, in both IJF and OJF tissues (Figure 5). However, DEGs and functional 
enriched terms in the OJF represented a possibility of biosynthesis of fatty acids, maybe in a unique pathway. More importantly, we came to a prediction based on the presence of the $A P O H$ gene in the OJF and the APP gene in the IJF. The metabolism of lipids in the outer jaw can be mainly mediated with the $A P O H$ protein as a transporter of lipids, and this is supported by other studies ${ }^{119,120}$. Meanwhile, APP might be involved in exchanging lipids from lower jawbone marrow fat and de novo synthesis within the IJF tissue. Specifically, enriched terms in the IJF support the creation of a new hypothesis: These fats may be able to transfer the receiving echolocation signals to the brain via neurons with the activation of neurotransmitters (Figure 6). Hence, APP might be an echolocation gene in toothed whales, but this still needs more research to be confirmed. The neural physiology of specific circuits used for echolocation in dolphins has evidenced in some studies ${ }^{121}$. Similar symptom of hemorrhaging has been observed in central nerve system (CNS) and acoustic jaw fat in beaked whales due to sonar ${ }^{122}$, therefore these could be the evidences to prove the link between IJFs and brain via the CNS for echolocation in toothed whales. Supporting, a study has described that presence of adipose tissue, blood vessel and nerves in the fibrous tissue of temporomandibular joint of dolphins play a role in echolocation as a neurological sensory function (McDonald et al., 2015; Bruno Cozzi; Stefan Huggenberger; Helmut Oelschläger, 2017). The involvement of neural system for acoustic motor-sensory coupling for echolocation has been investigated to human (Thaler, Arnott and Goodale, 2011; Flanagin et al., 2017) and bats (Covey, 2005; Razak and Fuzessery, 2015). The difference of DEGs between inner and OJFs may also correlate with the physiochemical difference of branched-chain lipid concentration and distribution among the two fats ${ }^{6}$. We believed that this is the first study of a transcriptomic analysis of mandibular/jaw/acoustic fats in Odontocetes (toothed whales). Therefore, there is still a big vacuum of knowledge to compare metabolism and biosynthesis of these specialized fats. We recommend further analyses of acoustic fat tissues of toothed whales using different omics tools in order to better understand the de novo synthesis of these unique lipids.

In summary, we performed a transcriptomic analysis on lower jaw fats of the Risso's dolphin as a representative organism of toothed whales that possess a special aquatic adaptation, their echolocation. During this study, the lower jaw fat of toothed whales has been extensively studied as a receiver of signals of echoes from a given object. However, the metabolism of these specialized fats is still largely unknown. According to a lipidomic analysis, these fats contain a combination of TAG and wax esters. The combination and distribution of these fats are different in the inner and outer lower jaw. We studied RNAs in both fats, and their DEGs were identified. Additionally, enrichment analyses were also conducted, mainly by Enricher at $p<0.05$. Our results indicated that $34 \mathrm{DEG}$ in OJF and nine DEGs in IJF were enriched. APOH, HNF4A, MYF6, SLC1A2, SLC2A2 and ALDOB were identified as key genes involved with lipid metabolism of functional enrichments. The main $\mathrm{GO}$ and pathway enrichments mainly suggested that these genes were positively related to lipoprotein lipase activities and the activation of pentose phosphate pathway in the OJF. On the other hand, IJF contains APP, DHX9, PXMP4 and THBS4 as highly expressed genes, which are responsible for lipid metabolism. These genes are mainly involved with the positive regulation of binding and amyloid-beta formation and the activation of ECM-receptor interactions. Our study also revealed the presence of wax ester synthase in both fat tissues and the 
possibility of fatty acid synthesis in IJF according to KEGG pathways. Moreover, we made a new hypothesis that IJF may directly transfer the receiving signals of echoes to the brain by potential activation of neurotransmitters by the APP gene; therefore, APP might be an echolocation gene. The interactions between IJFs and OJFs needs further investigation to reveal the underlying molecular mechanisms affecting the endogenous lipid metabolisms in toothed whales. These finding could be useful for understanding the industrialized biosynthesis of these specialized lipids. Therefore, more protection methods can be implemented for these animals and other conservation efforts.

\section{Declarations}

\section{Author contribution}

JDMS, YI, KY and SA designed the research. JDMS, RY, TS, NF and YI involved to perform the experimental procedures. JDMS, KY, SK, YI and SA contributed reagents and data analysis. JDMS wrote the main manuscript text. All authors reviewed the manuscript.

\section{Acknowledgements}

We would like to thank the Taiji Fishery Association, and researchers of National Research Institute of Far Seas Fisheries, Japan Fisheries Research and Education Agency for helping this research.

\section{Conflict of interest}

Authors do not have any conflict of interest.

\section{Additional information}

Supplementary File I

\section{References}

1. Litchfield, C. et al. Comparative lipid patterns in acoustical and nonacoustical fatty tissues of dolphins, porpoises and toothed whales. Comp. Biochem. Physiol. - Part B Biochem. (1975). doi:10.1016/0305-0491(75)90095-4

2. Koopman, H. N. Function and evolution of specialized endogenous lipids in toothed whales. J. Exp. Biol. 221, jeb161471 (2018).

3. Gabler, M. K., Gay, D. M., Westgate, A. J. \& Koopman, H. N. Microvascular characteristics of the acoustic fats: Novel data suggesting taxonomic differences between deep and shallow-diving odontocetes. J. Morphol. 279, 458-471 (2018).

4. Mooney, T. A., Yamato, M. \& Branstetter, B. K. Hearing in Cetaceans: From Natural History to Experimental Biology. Advances in Marine Biology 63, (Elsevier Ltd., 2012). 
5. Aroyan, J. L., Cranford, T. W., Kent, J. \& Norris, K. S. Computer modeling of acoustic beam formation in Delphinus delphis. J. Acoust. Soc. Am. 92, 2539-2545 (1992).

6. Koopman, H. N., Budge, S. M., Ketten, D. R. \& Iverson, S. J. Topographical distribution of lipids inside the mandibular fat bodies of odontocetes: Remarkable complexity and consistency. IEEE J. Ocean. Eng. 31, 95-106 (2006).

7. Trumble, S. J., Robinson, E. M., Berman-kowalewski, M., Potter, C. W. \& Usenko, S. Blue whale earplug reveals lifetime contaminant exposure and hormone pro fi les. 110, (2013).

8. Jung, J. L. et al. Qualitative and quantitative study of the highly specialized lipid tissues of cetaceans using HR-MAS NMR and classical GC. PLoS One 12, 1-23 (2017).

9. Wedmid, Y. et al. Heterogeneity of lipid composition within the cephalic melon tissue of the pilot whale (Globicephala melaena). Biochim. Biophys. Acta (BBA)/Lipids Lipid Metab. 326, 439-447 (1973).

10. Gatesy, J. et al. A phylogenetic blueprint for a modern whale. Mol. Phylogenet. Evol. 66, 479-506 (2013).

11. Koopman, H. N., Budge, S. M., Ketten, D. R. \& Iverson, S. J. Topographical distribution of lipids inside the mandibular fat bodies of odontocetes: Remarkable complexity and consistency. IEEE J. Ocean. Eng. 31, 95-106 (2006).

12. Sleos, J. C. \& Ackman, R. G. PID PATTERNS IN ACC AL FATTY TISSUES OF ES AND TOOTHED WH L AND INS ,. (1974).

13. Luo, L. \& Liu, M. Adipose tissue in control of metabolism. J. Endocrinol. 231, R77-R99 (2016).

14. Lanfranconi, M. P., Alvarez, A. F. \& Alvarez, H. M. Identification of genes coding for putative wax ester synthase/diacylglycerol acyltransferase enzymes in terrestrial and marine environments. $A M B$ Express 5, 1-13 (2015).

15. Ren, L. et al. Transcriptomic Analysis of the Regulation of Lipid Fraction Migration and Fatty Acid Biosynthesis in Schizochytrium sp. Sci. Rep. 7, 1-10 (2017).

16. Todd, E. V., Black, M. A. \& Gemmell, N. J. The power and promise of RNA-seq in ecology and evolution. Mol. Ecol. 25, 1224-1241 (2016).

17. Conesa, A. et al. A survey of best practices for RNA-seq data analysis. Genome Biol. 17, 1-19 (2016).

18. Schurch, N. J. et al. How many biological replicates are needed in an RNA-seq experiment and which differential expression tool should you use? Rna 22, 839-851 (2016).

19. Lamarre, S. et al. Optimization of an RNA-seq differential gene expression analysis depending on biological replicate number and library size. Front. Plant Sci. 9, (2018).

20. Ghosh, S. \& Chan, C. K. K. Analysis of RNA-seq data using TopHat and cufflinks. in Methods in Molecular Biology 1374, 339-361 (Humana Press Inc., 2016).

21. Koopman, H. N., Budge, S. M., Ketten, D. R. \& Iverson, S. J. Topographical Distribution of Lipids Inside the Mandibular Fat Bodies of Odontocetes: Remarkable Complexity and Consistency. 31, 95-106 (2006). 
22. Ackman, R. G., Sipos, J. C., Eaton, C. A., Hilaman, B. L. \& Litchfield, C. Molecular species of wax esters in jaw fat of atlantic bottlenose dolphin, Tursiops truncatus. Lipids 8, 661-667 (1973).

23. Ames, A. L., Van Vleet, E. S. \& Reynolds, J. E. Comparison of lipids in selected tissues of the Florida manatee (Order Sirenia) and bottlenose dolphin (Order Cetacea; Suborder Odontoceti). Comp. Biochem. Physiol. - B Biochem. Mol. Biol. 132, 625-634 (2002).

24. Morii, H. \& Kaneda, T. Biosynthesis of branched-chain fatty acids from branched-chain amino acids in subcutaneous tissue of the marine little toothed whale, Stenella caeruleo-alba. Comp. Biochem. Physiol. - Part B Biochem. 71, 357-365 (1982).

25. Michal, J. J., Zhang, Z. W., Gaskins, C. T. \& Jiang, Z. The bovine fatty acid binding protein 4 gene is significantly associated with marbling and subcutaneous fat depth in Wagyu x Limousin F2 crosses. Anim. Genet. 37, 400-402 (2006).

26. Leduc, M. S. et al. Comprehensive evaluation of apolipoprotein $\mathrm{H}$ gene (APOH) variation identifies novel associations with measures of lipid metabolism in GENOA. J. Lipid Res. 49, 2648-2656 (2008).

27. Jin, S. et al. Expression of genes related to lipid transport in meat-type ducks divergent for low or high residual feed intake. Asian-Australasian J. Anim. Sci. 33, 416-423 (2020).

28. Bu, P. et al. Aldolase B-Mediated Fructose Metabolism Drives Metabolic Reprogramming of Colon Cancer Liver Metastasis. Cell Metab. 27, 1249-1262.e4 (2018).

29. Laity, J. H., Lee, B. M. \& Wright, P. E. Zinc finger proteins: New insights into structural and functional diversity. Current Opinion in Structural Biology 11, 39-46 (2001).

30. Li, G., Ma, X. \& Xu, L. The roles of zinc finger proteins in non-alcoholic fatty liver disease. Liver Res. 4, 35-39 (2020).

31. Chu, X. et al. A retrospective case control study identifies peripheral blood mononuclear cell albumin RNA expression as a biomarker for non-alcoholic fatty liver disease. Langenbeck's Arch. Surg. 1-8 (2019). doi:10.1007/s00423-019-01848-0

32. Das, U. N. Saturated Fatty Acids, MUFAs and PUFAs Regulate Ferroptosis. Cell Chem. Biol. 26, 309311 (2019).

33. Yen, M. C. et al. New insight on solute carrier family 27 member 6 (SLC27a6) in tumoral and nontumoral breast cells. Int. J. Med. Sci. 16, 366-375 (2019).

34. Xu, X. et al. Effects of dietary apple polyphenol supplementation on carcass traits, meat quality, muscle amino acid and fatty acid composition in finishing pigs. Food Funct. 10, 7426-7434 (2019).

35. Khristi, V. et al. Liver transcriptome data of Esr1 knockout male rats reveals altered expression of genes involved in carbohydrate and lipid metabolism. Data Br. 22, 771-780 (2019).

36. Wisely, G. B. et al. Hepatocyte nuclear factor 4 is a transcription factor that constitutively binds fatty acids. Structure 10, 1225-1234 (2002).

37. Chan, J., Nakabayashi, H. \& Wong, N. C. W. HNF-4 increases activity of the rat Apo A1 gene. Nucleic Acids Res. 21, 1205-1211 (1993). 
38. Chen, Z., Gropler, M. C., Mitra, M. S. \& Finck, B. N. Complex Interplay between the Lipin 1 and the Hepatocyte Nuclear Factor 4 a (HNF4a) Pathways to Regulate Liver Lipid Metabolism. PLoS One 7, 1-10 (2012).

39. Xia, W. F. et al. Swedish mutant APP suppresses osteoblast differentiation and causes osteoporotic deficit, which are ameliorated by N-acetyl-L-cysteine. J. Bone Miner. Res. 28, 2122-2135 (2013).

40. Puig, K. L. et al. Amyloid precursor protein modulates macrophage phenotype and diet-dependent weight gain. Sci. Rep. 7, 1-14 (2017).

41. Zhang, Q. et al. Promoting therapeutic angiogenesis of focal cerebral ischemia using thrombospondin-4 (TSP4) gene-modified bone marrow stromal cells (BMSCs) in a rat model. J. Transl. Med. 17, 1-13 (2019).

42. Dashnamoorthy, R. et al. Lipid Addiction of Diffuse Large B-Cell Lymphoma (DLBCL): Fatty Acid Synthase (FASN) and PI3K Dependent Cell Death Identifies a Novel Therapeutic Paradigm. Blood 126, 1284-1284 (2015).

43. Grimm, M. O. W., Mett, J., Grimm, H. S. \& Hartmann, T. App function and lipids: A bidirectional link. Front. Mol. Neurosci. 10, 1-18 (2017).

44. Kuleshov, M. V. et al. Enrichr: a comprehensive gene set enrichment analysis web server 2016 update. Nucleic Acids Res. 44, W90-W97 (2016).

45. Weichselbaum, D., Zagrovic, B. \& Polyansky, A. A. Fuento: functional enrichment for bioinformatics. Bioinformatics 33, 2604-2606 (2017).

46. Wadi, L., Meyer, M., Weiser, J., Stein, L. D. \& Reimand, J. Impact of outdated gene annotations on pathway enrichment analysis. Nat. Methods 13, 705-706 (2016).

47. Dymond, M. K. Mammalian phospholipid homeostasis: Evidence that membrane curvature elastic stress drives homeoviscous adaptation in vivo. J. R. Soc. Interface 13, (2016).

48. Zhao, W. S. et al. Lipoprotein lipase, tissue expression and effects on genes related to fatty acid synthesis in goat mammary epithelial cells. Int. J. Mol. Sci. 15, 22757-22771 (2014).

49. Mattijssen, F. \& Kersten, S. Regulation of triglyceride metabolism by Angiopoietin-like proteins. Biochimica et Biophysica Acta - Molecular and Cell Biology of Lipids 1821, 782-789 (2012).

50. Kershaw, E. E. et al. PPARy regulates adipose triglyceride lipase in adipocytes in vitro and in vivo. Am. J. Physiol. - Endocrinol. Metab. 293, 1736-1745 (2007).

51. Muscat, G. E. O. et al. Regulation of cholesterol homeostasis and lipid metabolism in skeletal muscle by liver X receptors. J. Biol. Chem. 277, 40722-40728 (2002).

52. Ouweneel, A. B., Thomas, M. J. \& Sorci-Thomas, M. G. The ins and outs of lipid rafts: Functions in intracellular cholesterol homeostasis, microparticles, and cell membranes Running title: lipid rafts in intracellular membranes and extracellular vesicles. (2020).

53. Yen, C. L. E., Brown IV, C. H., Monetti, M. \& Farese, R. V. A human skin multifunctional Oacyltransferase that catalyzes the synthesis of acylglycerols, waxes, and retinyl esters. J. Lipid Res. 46, 2388-2397 (2005). 
54. Ruan, H. \& Pownall, H. J. Overexpression of 1-acyl-glycerol-3-phosphate acyltransferase-a enhances lipid storage in cellular models of adipose tissue and skeletal muscle. Diabetes 50, 233-240 (2001).

55. Jenkins, C. M., Yang, J., Sims, H. F. \& Gross, R. W. Reversible high affinity inhibition of phosphofructokinase-1 by Acyl-CoA: A mechanism integrating glycolytic flux with lipid metabolism. J. Biol. Chem. 286, 11937-11950 (2011).

56. Ackerman, D. et al. Triglycerides Promote Lipid Homeostasis during Hypoxic Stress by Balancing Fatty Acid Saturation. Cell Rep. 24, 2596-2605.e5 (2018).

57. Bruss, M. L. Lipids and Ketones. in Clinical Biochemistry of Domestic Animals 81-115 (Elsevier Inc., 2008). doi:10.1016/B978-0-12-370491-7.00004-0

58. Ivanova, E. A., Myasoedova, V. A., Melnichenko, A. A., Grechko, A. V. \& Orekhov, A. N. Small Dense Low-Density Lipoprotein as Biomarker for Atherosclerotic Diseases. Oxid. Med. Cell. Longev. 2017, (2017).

59. Kusunoki, M. et al. The lipoprotein lipase activator, NO-1886, suppresses fat accumulation and insulin resistance in rats fed a high-fat diet. Diabetologia 43, 875-880 (2000).

60. Wasylenko, T. M., Ahn, W. S. \& Stephanopoulos, G. The oxidative pentose phosphate pathway is the primary source of NADPH for lipid overproduction from glucose in Yarrowia lipolytica. Metab. Eng. 30, 27-39 (2015).

61. Zhao, L. et al. Role of pentose phosphate pathway in lipid accumulation of oleaginous fungus Mucor circinelloides. RSC Adv. 5, 97658-97664 (2015).

62. Rausa, F. M. et al. Elevated Levels of Hepatocyte Nuclear Factor $3 \beta$ in Mouse Hepatocytes Influence Expression of Genes Involved in Bile Acid and Glucose Homeostasis. Mol. Cell. Biol. 20, 8264-8282 (2000).

63. Rui, L. Energy Metabolism in the Liver. (2014). doi:10.1002/cphy.c130024

64. Burant, C. F. Chapter 2 Facilitative glucose transport. Princ. Med. Biol. 4, 67-86 (1996).

65. Mueller, E. Understanding the variegation of fat: Novel regulators of adipocyte differentiation and fat tissue biology. Biochim. Biophys. Acta - Mol. Basis Dis. 1842, 352-357 (2014).

66. Miettinen, S., Sarkanen, J. R. \& Ashammakhi, N. Adipose Tissue and Adipocyte Differentiation: Molecular and Cellular Aspects and Tissue Engineering Applications. Top. Tissue Eng. 4, 1-26 (2008).

67. Dixon, D. P. \& Edwards, R. Selective binding of glutathione conjugates of fatty acid derivatives by plant glutathione transferases. J. Biol. Chem. 284, 21249-21256 (2009).

68. Sun, S., Li, H., Chen, J. \& Qian, Q. Lactic acid: No longer an inert and end-product of glycolysis. Physiology 32, 453-463 (2017).

69. Reese-Wagoner, A., Thompson, J. \& Banaszak, L. Structural properties of the adipocyte lipid binding protein. Biochimica et Biophysica Acta - Molecular and Cell Biology of Lipids 1441, 106-116 (1999).

70. Bernlohr, D. A., Simpson, M. A., Hertzel, A. V. \& Banaszak, L. J. INTRACELLULAR LIPID-BINDING. 277303 (1997). 
71. Grösgen, S., Grimm, M. O. W., Frieß, P. \& Hartmann, T. Role of amyloid beta in lipid homeostasis. Biochimica et Biophysica Acta - Molecular and Cell Biology of Lipids 1801, 966-974 (2010).

72. Thompson, J. C. et al. Serum amyloid A3 is pro-atherogenic. Atherosclerosis 268, 32-35 (2018).

73. Hu, N. et al. The relationship between blood lipids and plasma amyloid beta is depend on blood pressure: a population-based cross-sectional study. Lipids Health Dis. 19, 8 (2020).

74. Zeng, X. et al. Erratum: Oleic acid ameliorates palmitic acid induced hepatocellular lipotoxicity by inhibition of ER stress and pyroptosis (Nutrition and Metabolism (2020) 17 (11) DOI: 10.1186/s12986-020-0434-8). Nutr. Metab. 17, 1-14 (2020).

75. Laha, A., Majumder, A., Singh, M. \& Tyagi, S. C. Connecting homocysteine and obesity through pyroptosis, gut microbiome, epigenetics, peroxisome proliferator-activated receptor $\gamma$, and zinc finger protein 407. Can. J. Physiol. Pharmacol. 96, 971-976 (2018).

76. Welte, M. A. Fat on the move: Intracellular motion of lipid droplets. Biochem. Soc. Trans. 37, 991-996 (2009).

77. Welte, M. A. Bidirectional transport along microtubules. Curr. Biol. 14, 525-537 (2004).

78. Hubler, M. J. \& Kennedy, A. J. Role of lipids in the metabolism and activation of immune cells. J. Nutr. Biochem. 34, 1-7 (2016).

79. Finlay, D. \& Cantrell, D. A. Metabolism, migration and memory in cytotoxic T cells. Nat. Rev. Immunol. 11, 109-117 (2011).

80. Sharabi, A. \& Tsokos, G. C. T cell metabolism: new insights in systemic lupus erythematosus pathogenesis and therapy. Nat. Rev. Rheumatol. 16, 100-112 (2020).

81. Boeri, L., Albani, D., Raimondi, M. T. \& Jacchetti, E. Mechanical regulation of nucleocytoplasmic translocation in mesenchymal stem cells: characterization and methods for investigation. Biophys. Rev. 11, 817-831 (2019).

82. Kis, V., Barti, B., Lippai, M. \& Sass, M. Specialized Cortex Glial Cells Accumulate Lipid Droplets in Drosophila melanogaster. PLoS One 10, e0131250 (2015).

83. Barber, C. N. \& Raben, D. M. Lipid metabolism crosstalk in the brain: Glia and neurons. Front. Cell. Neurosci. 13, 1-5 (2019).

84. Guy, A. T. et al. Glycerophospholipid regulation of modality-specific sensory axon guidance in the spinal cord. Science (80-. ). 349, 974-977 (2015).

85. Nave, K. A., Tzvetanova, I. D. \& Schirmeier, S. Glial Cell Evolution: The Origins of a Lipid Store. Cell Metab. 26, 701-702 (2017).

86. Cordero-Morales, J. F. \& Vásquez, V. How Lipids Contribute to lon Channel Function, a Fat Perspective on Direct and Indirect Interactions. doi:10.1016/j.sbi.2018.03.015

87. Cockcroft, S. \& Raghu, P. Phospholipid transport protein function at organelle contact sites. Curr. Opin. Cell Biol. 53, 52-60 (2018).

88. Yang, Y., Lee, M. \& Fairn, G. D. Phospholipid subcellular localization and dynamics. J. Biol. Chem. 293, 6230-6240 (2018). 
89. Lodhi, I. J. \& Semenkovich, C. F. Peroxisomes: A nexus for lipid metabolism and cellular signaling. Cell Metabolism 19, 380-392 (2014).

90. Kong, J. et al. Spatiotemporal contact between peroxisomes and lipid droplets regulates fastinginduced lipolysis via PEX5. Nat. Commun. 11, 1-16 (2020).

91. Kohlwein, S. D., Veenhuis, M. \& van der Klei, I. J. Lipid droplets and peroxisomes: Key players in cellular lipid homeostasis or a matter of fat-store'em up or burn'em down. Genetics 193, 1-50 (2013).

92. Joshi, A. S. \& Cohen, S. Lipid droplet and peroxisome biogenesis: Do they go hand-in-hand? Front. Cell Dev. Biol. 7, 1-9 (2019).

93. Powell, M. J. Phylogenetic implications of the microbody-lipid globule complex in zoosporic fungi. BioSystems 10, 167-180 (1978).

94. Bankaitis, V. A., Garcia-Mata, R. \& Mousley, C. J. Golgi membrane dynamics and lipid metabolism. Curr. Biol. 22, R414-R424 (2012).

95. Munro, S. Lipid Rafts: Elusive or Illusive? Cel/ 115, 377-388 (2003).

96. Vance, J. E., Campenot, R. B. \& Vance, D. E. The synthesis and transport of lipids for axonal growth and nerve regeneration. Biochimica et Biophysica Acta - Molecular and Cell Biology of Lipids 1486, 84-96 (2000).

97. Posse de Chaves, E. I., Rusinol, A. E., Vance, D. E., Campenot, R. B. \& Vance, J. E. Role of lipoproteins in the delivery of lipids to axons during axonal regeneration. J. Biol. Chem. 272, 30766-30773 (1997).

98. Rodríguez-Berdini, L. \& Caputto, B. L. Lipid metabolism in neurons: A brief story of a novel c-Fosdependent mechanism for the regulation of their synthesis. Front. Cell. Neurosci. 13, 1-9 (2019).

99. Yang, C. et al. Rewiring Neuronal Glycerolipid Metabolism Determines the Extent of Axon Regeneration. Neuron 105, 276-292.e5 (2020).

100. Leitinger, B. \& Hogg, N. The involvement of lipid rafts in the regulation of integrin function. J. Cell Sci. $115,963-972$ (2002).

101. Cooper, G. M. The Nuclear Envelope and Traffic between the Nucleus and Cytoplasm. (2000).

102. Merta, H. \& Bahmanyar, S. The Inner Nuclear Membrane Takes On Lipid Metabolism. Dev. Cell 47, 397-399 (2018).

103. Romanauska, A. \& Köhler, A. The Inner Nuclear Membrane Is a Metabolically Active Territory that Generates Nuclear Lipid Droplets. Cell 174, 700-715.e18 (2018).

104. Roy, K. \& Marin, E. P. Lipid Modifications in Cilia Biology. J. Clin. Med. 8, 921 (2019).

105. Mariman, E. C. M. \& Wang, P. Adipocyte extracellular matrix composition, dynamics and role in obesity. Cell. Mol. Life Sci. 67, 1277-1292 (2010).

106. Lee, H. J. et al. Comparative Transcriptome Analysis of Adipose Tissues Reveals that ECM-Receptor Interaction Is Involved in the Depot-Specific Adipogenesis in Cattle. PLoS One 8, (2013).

107. Song, C. et al. RNA-Seq Analysis Identifies Differentially Expressed Genes in Subcutaneous Adipose Tissue in Qaidaford Cattle, Cattle-Yak, and Angus Cattle. Animals 9, 1077 (2019). 
108. Cui, H., Zheng, M., Zhao, G., Liu, R. \& Wen, J. Identification of differentially expressed genes and pathways for intramuscular fat metabolism between breast and thigh tissues of chickens. BMC Genomics 19, 1-9 (2018).

109. Bakhtiarizadeh, M. R., Salehi, A., Alamouti, A. A., Abdollahi-Arpanahi, R. \& Salami, S. A. Deep transcriptome analysis using RNA-Seq suggests novel insights into molecular aspects of fat-tail metabolism in sheep. Sci. Rep. 9, 1-14 (2019).

110. Islinger, M., Voelkl, A., Fahimi, H. D. \& Schrader, M. The peroxisome: an update on mysteries 2.0. Histochem. Cell Biol. 150, 443-471 (2018).

111. Postila, P. A. \& Róg, T. A Perspective: Active Role of Lipids in Neurotransmitter Dynamics. Mol. Neurobiol. 57, 910-925 (2020).

112. Borroni, M. V., Vallés, A. S. \& Barrantes, F. J. The lipid habitats of neurotransmitter receptors in brain. Biochim. Biophys. Acta - Biomembr. 1858, 2662-2670 (2016).

113. Wyler, S. C., Lord, C. C., Lee, S., Elmquist, J. K. \& Liu, C. Serotonergic control of metabolic homeostasis. Front. Cell. Neurosci. 11, 1-9 (2017).

114. Morrell, A., Tallino, S., Yu, L. \& Burkhead, J. L. The role of insufficient copper in lipid synthesis and fatty-liver disease. IUBMB Life 69, 263-270 (2017).

115. Yang, H. et al. Copper-dependent amino oxidase 3 governs selection of metabolic fuels in adipocytes. PLoS Biol. 16, 1-27 (2018).

116. Younessi, P. \& Yoonessi, A. Advanced glycation end-products and their receptor-mediated roles: Inflammation and oxidative stress. Iran. J. Med. Sci. 36, 154-166 (2011).

117. Ott, C. et al. Role of advanced glycation end products in cellular signaling. Redox Biol. 2, 411-429 (2014).

118. Takino, J. ichi, Nagamine, K., Hori, T., Sakasai-Sakai, A. \& Takeuchi, M. Contribution of the toxic advanced glycation end-products-receptor axis in nonalcoholic steatohepatitis-related hepatocellular carcinoma. World J. Hepatol. 7, 2459-2469 (2015).

119. Leduc, M. S. et al. Comprehensive evaluation of apolipoprotein $\mathrm{H}$ gene ( $\mathrm{APOH})$ variation identifies novel associations with measures of lipid metabolism in GENOA. 49, (2008).

120. Erkkilä, A. \& Lankinen, M. Fish and fish oil and lipoprotein particle number and size. in Fish and Fish Oil in Health and Disease Prevention 239-247 (Elsevier Inc., 2016). doi:10.1016/B978-0-12-8028445.00022-1

121. Fulton, J. Dolphin Biosonar Echolocation A Case Study. Process. Biol. Hear. 203-216 (2007).

122. Martín, V. et al. "Gas and Fat Embolic Syndrome" Involving a Mass Stranding of Beaked Whales (Family Ziphiidae) Exposed to Anthropogenic Sonar Signals. Vet. Pathol. 42, 446-457 (2005).

123. View, M. RNA clean-up User manual. (2014).

124. Martinez-Viaud, K. A. et al. New de novo assembly of the Atlantic bottlenose dolphin (Tursiops truncatus) improves genome completeness and provides haplotype phasing. Gigascience 8, 1-9 (2019). 
125. Schmieder, R. \& Edwards, R. Quality control and preprocessing of metagenomic datasets. Bioinformatics 27, 863-864 (2011).

126. Kim, D., Paggi, J. M., Park, C., Bennett, C. \& Salzberg, S. L. Graph-based genome alignment and genotyping with HISAT2 and HISAT-genotype. Nat. Biotechnol. 37, 907-915 (2019).

127. Pertea, M. et al. StringTie enables improved reconstruction of a transcriptome from RNA-seq reads. Nat. Biotechnol. 33, 290-295 (2015).

128. Trapnell, C. et al. Transcript assembly and quantification by RNA-Seq reveals unannotated transcripts and isoform switching during cell differentiation. Nat. Biotechnol. 28, 511-515 (2010).

129. Frazee, A. C. et al. Ballgown bridges the gap between transcriptome assembly and expression analysis. Nat. Biotechnol. 33, 243-246 (2015).

130. Robinson, M. D., McCarthy, D. J. \& Smyth, G. K. edgeR: A Bioconductor package for differential expression analysis of digital gene expression data. Bioinformatics 26, 139-140 (2009).

131. Trapnell, C. et al. Differential analysis of gene regulation at transcript resolution with RNA-seq. Nat. Biotechnol. 31, 46-53 (2013).

132. Kuleshov, M. V. et al. Enrichr: a comprehensive gene set enrichment analysis web server 2016 update. Nucleic Acids Res. 44, W90-W97 (2016).

133. Montojo, J., Zuberi, K., Rodriguez, H., Bader, G. D. \& Morris, Q. GeneMANIA: Fast gene network construction and function prediction for Cytoscape. F1000Research 3, 2-8 (2014).

134. Takara Bio Inc. RNAiso Plus ( Total RNA extraction reagent ) Product Manual. (2017).

135. Takara Bio Inc. RR420A SYBR ${ }^{\circledR}$ Premix Ex Taq ${ }^{\text {TM }}$ ( Tli RNaseH Plus ) Product Manual. (2015).

\section{Figures}


A

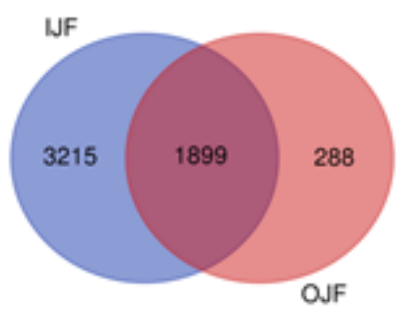

$\mathrm{C}$

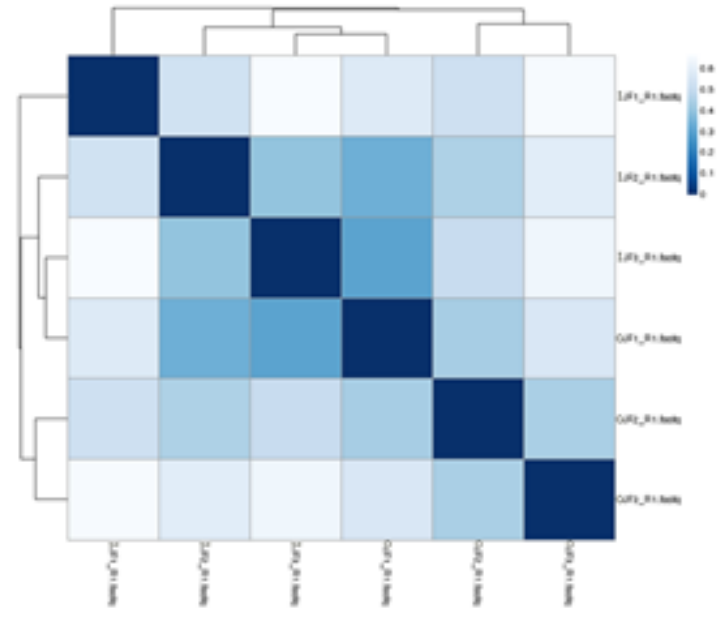

B

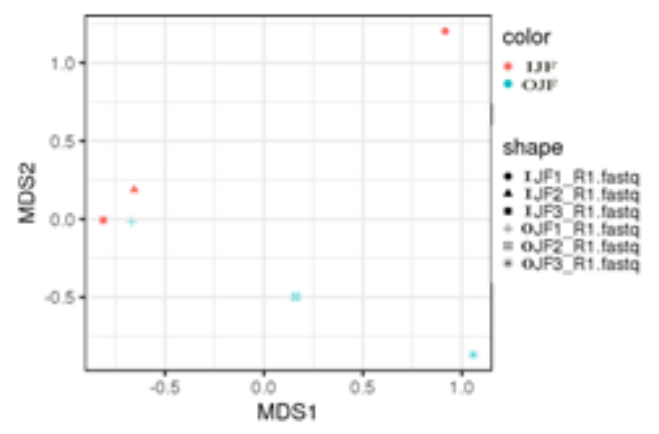

D

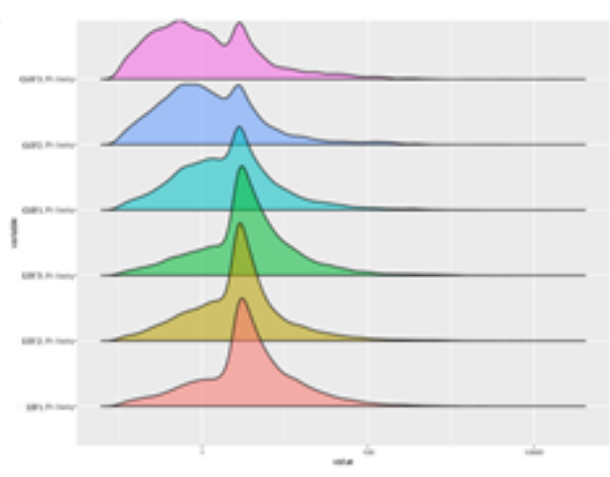

\section{Figure 1}

Distribution of genes among libraries and two jaw fat types. A; Ven diagram of distribution of genes in IJF and OJF with equal or more than 1 FPKM value. B; Multi-dimensional scaling plot of detailed log 10 distribution in three replicates and two fat tissues. C; Distribution of sample replicates in two different fat tissues. D; Histogram of gene counts in different replicates of IJF and OJF.

A

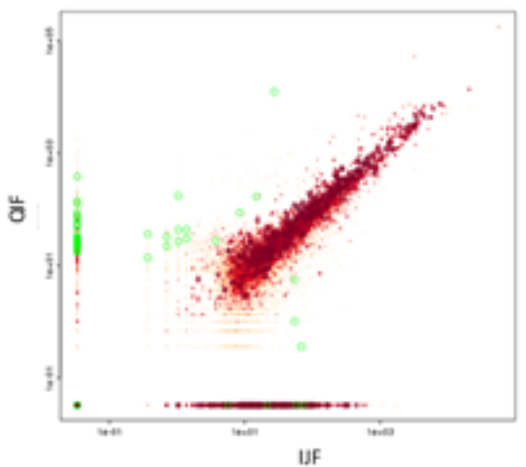

B

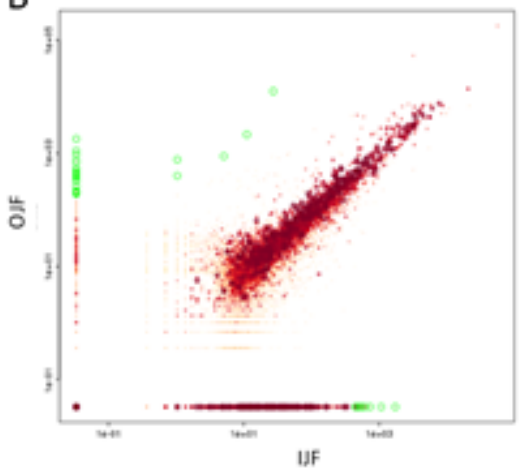

C

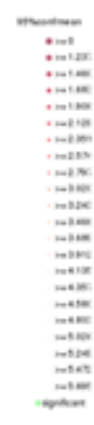

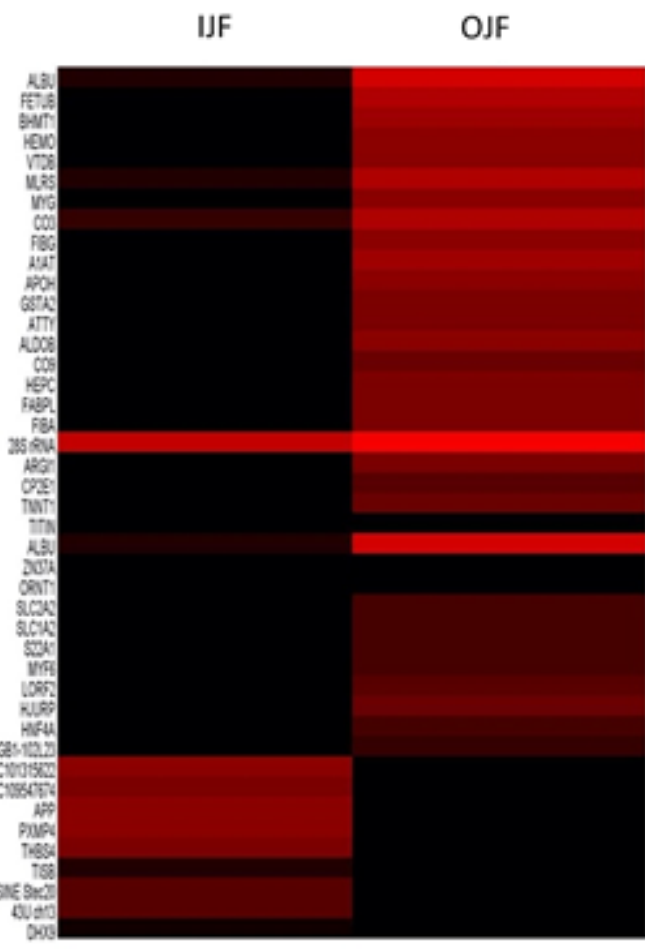


Figure 2

Distribution of differentially-expressed genes among IJF and OJF. A; Scatter plot by edgeR. B; Scatter plot by Cuffdiff. C; Heat map of highly-expressed genes in inner and outer jaw fats based on DEGs of edgeR and Cuffdiff statistics.

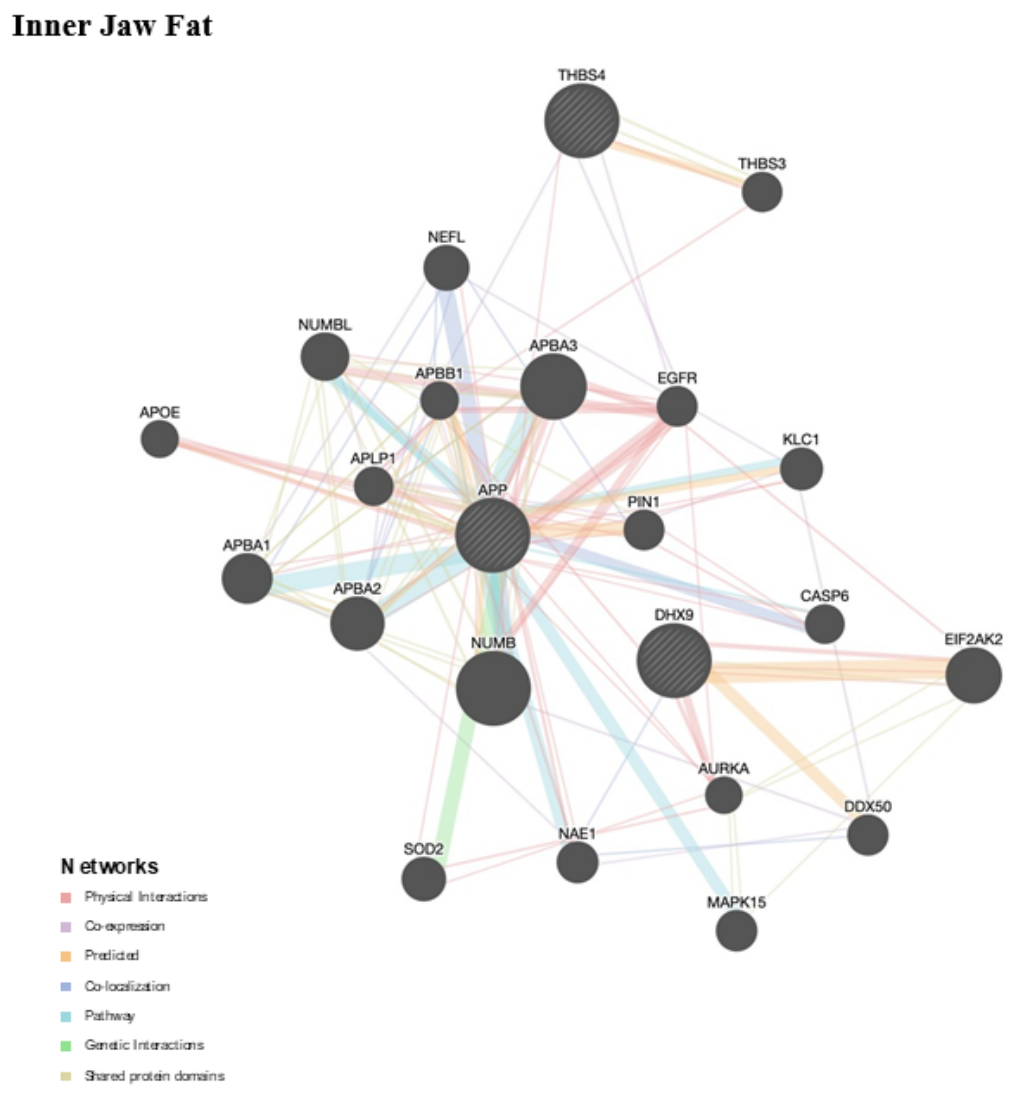

\section{Outer Jaw Fat}

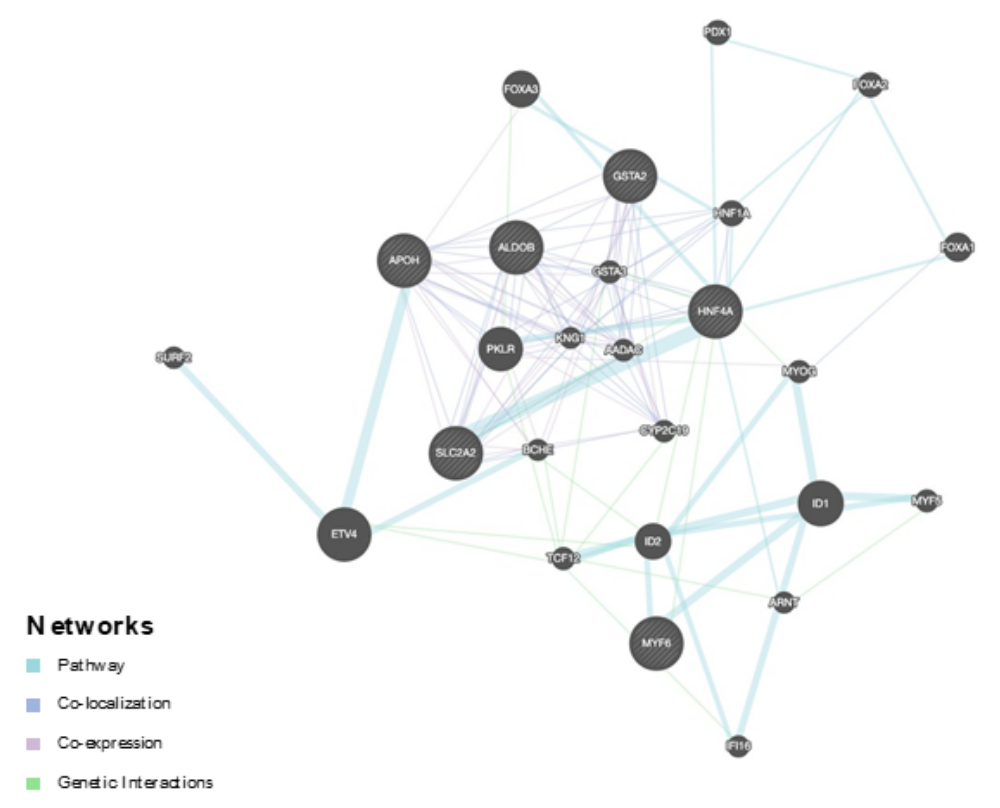

Figure 3 
Gene interaction networks by GeneMANIA analysis

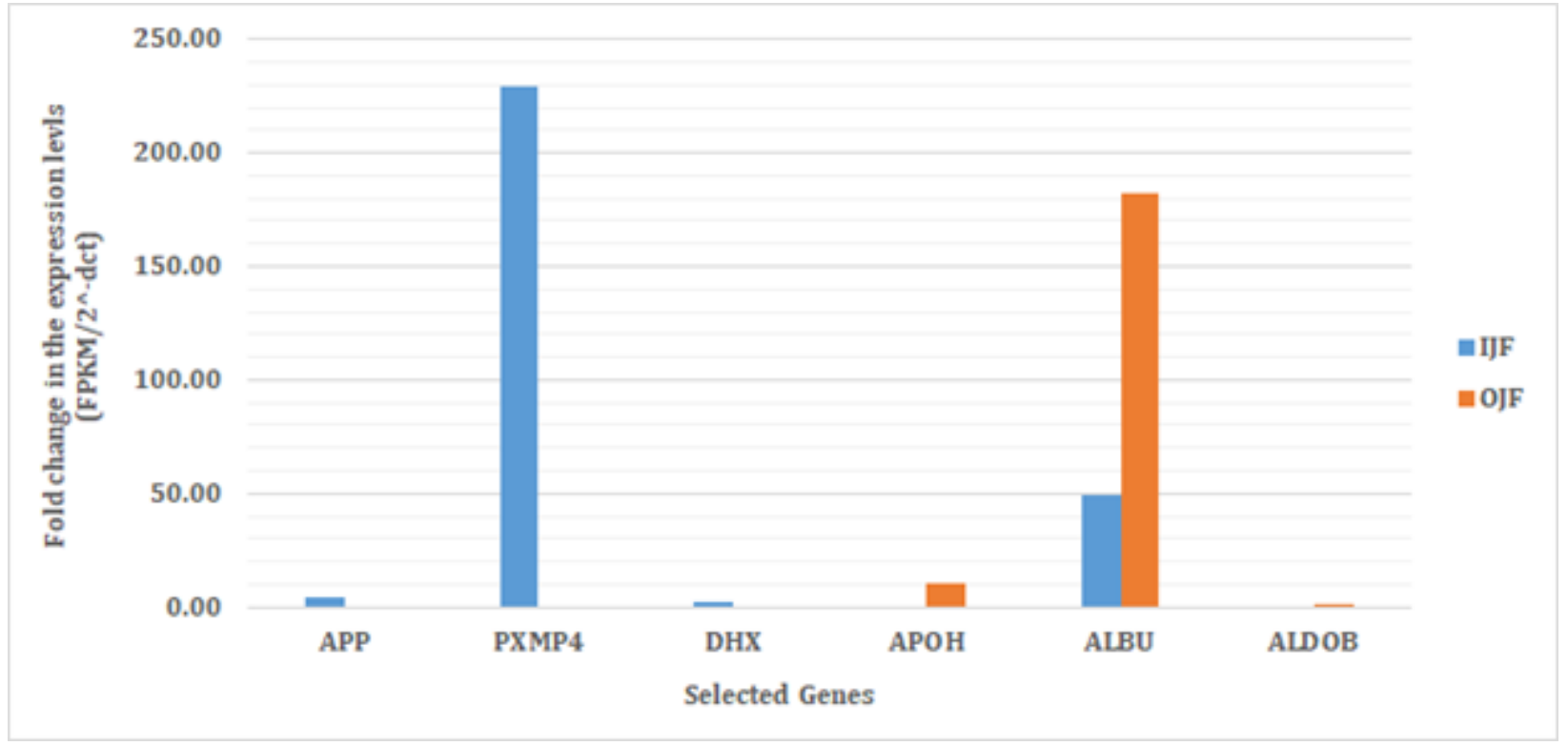

Figure 4

Validation of the differential expression genes in the OJF and IJF. Selected genes in the X axis with values of fold change (FC) and fragments per kilobase of exon model per million reads mapped (FPKM) vs relative percentage in the $\mathrm{Y}$ axis.

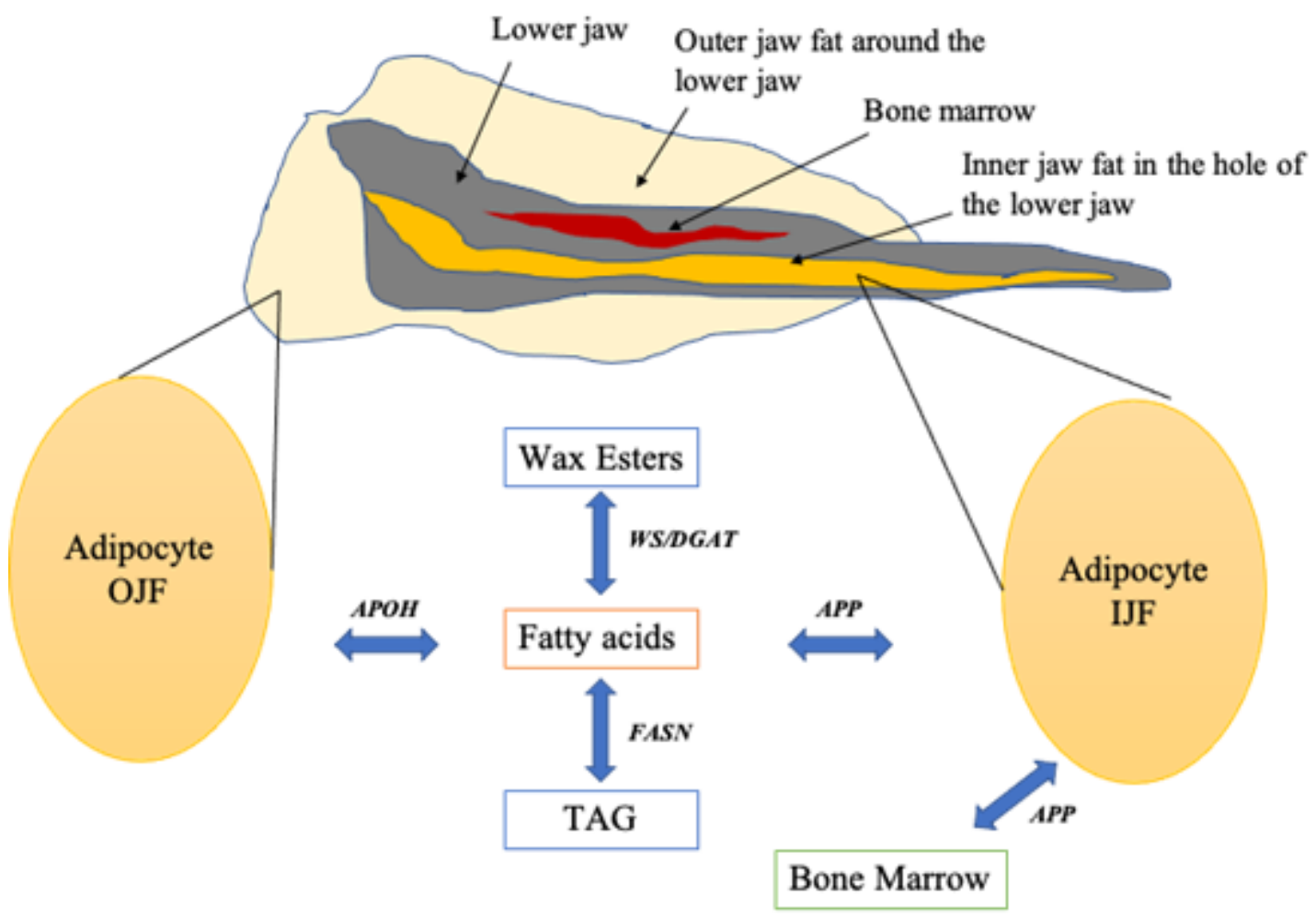

Figure 5

Predicted lipid metabolism in outer and inner jaw fats in toothed whales 


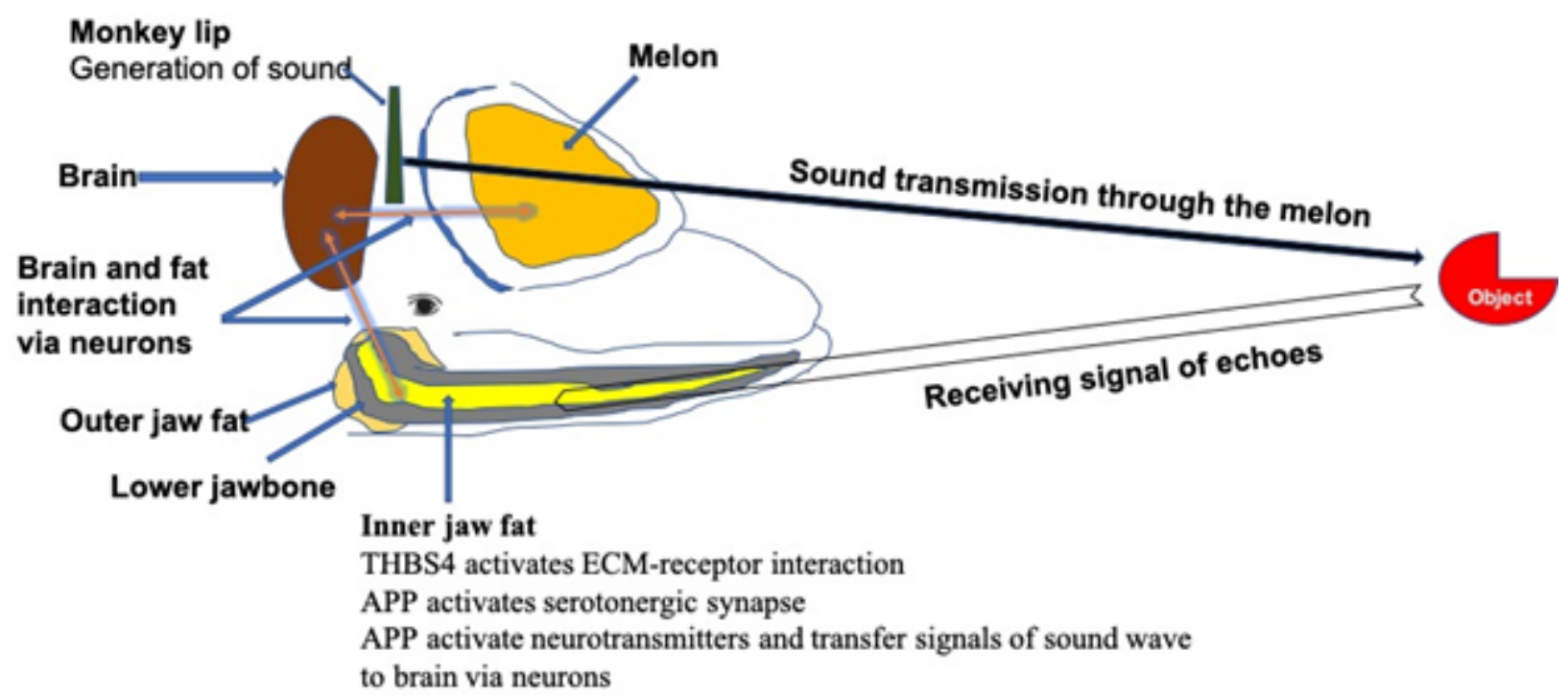

Figure 6

Predicted new hypothesis for echolocation between sound signal and brain via inner jaw fat

\section{Supplementary Files}

This is a list of supplementary files associated with this preprint. Click to download.

- SupplementaryFilesSR21I.pdf 